\title{
Evolution en cours de culture de la qualité d'hôte de trois espèces de ray-grass vis-à-vis du nématode Meloidogyne naasi Franklin
}

\author{
Françoise PERSON-DEDRYVER \\ avec la collaboration technique de Danielle PANNETIER \\ I.N.R.A., Laboratoire de Zoologie, Centre de Recherches de Rennes, F 35650 Le Rheu
}

RÉSUMÉ

\begin{abstract}
Une expérimentation conduite durant 19 mois sur du sol naturellement infesté par Meloidogyne naasi a permis une étude comparative de l'évolution de la qualité d'hôte de variétés de 3 espèces de ray-grass en cours de végétation.

En se référant aux nombres de femelles et d'œufs du nématode produits par $\mathrm{g}$ de racine les ray-grass italiens, variétés les moins persistantes de l'essai, restent durant toute l'expérience, les meilleurs hôtes. Ils favorisent un développement rapide en femelles. Les 3 ray-grass hybrides et le ray-grass anglais cv. Réveillé forment plus de femelles et d'œufs du nématode par $\mathrm{g}$ de racine que les cultivars de ray-grass anglais cvs Vigor et GrasslandsRuani. La variété $\mathrm{cv}$. Grasslands-Ruani peut être considérée comme hôte très médiocre pour ce parasite. La fécondité des femelles reste, durant toute la période d'essai, statistiquement identique pour la plupart des cultivars. La différence enregistrée dans le nombre d'œufs formés par $\mathrm{g}$ de racine entre espèces et variétés provient essentiellement du nombre de femelles qui se sont développées sur les différents cultivars.

Il y a 2 générations annuelles du parasite sur ray-grass, la $1^{r e}$ en juin-juillet, la $2^{e}$, soit en automne, soit en début de période hivernale. Cette seconde génération présente des effectifs de nématodes beaucoup plus importants que la première.
\end{abstract}

Mots clés additionnels : Graminées, Lolium spp., développement, résistance variétale, dynamique de population. ryegrass species.

The host-range of ryegrass species for Meloidogyne naasi has been studied in experiments in pots filled with infested soil. During the 19 months of experiment, the Italian ryegrasses, that had the lowest degree of perenniality, gave the highest number of females or eggs of the nematode per $\mathrm{g}$ of root. At low infestation level, a considerable number of females appeared on these Italian ryegrasses. Increased numbers of females and eggs were produced on 3 hybrid ryegrasses and the perennial ryegrass $\mathrm{cv}$. Réveillé, more than on perennial ryegrass cvs Vigor and Grasslands-Ruani. Cv. Grasslands-Ruani was a bad host for the nematode.

Female fecundity was statistically similar on a large number of cultivars tested. The different numbers of eggs produced per $\mathrm{g}$ of root and observed between species and varieties were the consequence of different development of females on these cultivars.

This study showed that 2 generations of the nematode could develop on ryegrasses, the 1st in June and July, and the second in autumn or at the beginning of winter. Numbers of females or eggs produced per $g$ of root were maximum in autumn with the second generation of $M$. naasi.

Additional key words : Gramineae, Lolium spp., development, resistance of variety, population, dynamics.

\section{INTRODUCTION}

Les ray-grass sont réputés être parmi les graminées fourragères, les meilleurs hôtes de $M$. naasi. Les raygrass italiens, qui présentent une durée de culture limitée comparée à celles des ray-grass anglais et hybride, sont ceux qui multiplient le plus le nématode jusqu'à la 2e coupe (COOK \& YORK, 1979 ; COOK et al., 1980 ; PERSON-DEDRYVER \& FISCHER, 1987). La dynamique des populations endoradiculaires formées chez chaque espèce n'est pas connue au-delà de 4 à 5 mois de culture. Seules quelques références concernent les popula- 
tions trouvées dans le sol et prouvent que le nématode se multiplie toujours beaucoup sur ray-grass (COOK \& YORK, 1983 ; GOORIS \& D'HERDE, 1977). Il est cependant intéressant de savoir si, en cours de culture, les qualités d'hôte des 3 espèces de ray-grass ne vont pas se modifier et s'inverser en fonction de leurs pérennités différentes.

Un essai a donc été mis en place, sur sol naturellement infesté pour suivre l'évolution des populations endoradiculaires de $M$. naasi sur 10 variétés appartenant à 3 espèces de ray-grass, dont les qualités intrinsèques d'hôte ont déjà fait l'objet d'une note (PERSONDEDRYVER \& FISCHER, 1987). Deux variétés de raygrass anglais cvs Vigor et Grasslands-Ruani, présentant un caractère hôte très médiocre pour le nématode (PERSON-DEDRYVER \& FisCHER, 1987), ont été introduites dans l'essai pour vérifier que leur mauvaise qualité d'hôte n'est pas affectée par le vieillissement de la culture. Cette étude est nécessaire, si l'on veut proposer l'emploi de graminées hôtes médiocres dans les rotations pour éviter les dégâts sur les cultures suivantes et plus particulièrement les céréales à paille (COOK \& YORK, 1979).

La qualité d'hôte des ray-grass est évaluée après comptage des femelles formées dans les racines. Ces femelles pondent, dans les galles induites, des œufs contenant des larves au $2^{\mathrm{e}}$ stade de leur développement (L2), qui sont dites «enchorionnées ». Les L2 peuvent alors, soit entrer en diapause, soit sortir de l'œuf et devenir immédiatement infectieuses. Le pouvoir multiplicateur des ray-grass vis-à-vis du nématode sera reflété par le nombre d'œufs trouvés dans les racines, dépendant de la fécondité et du nombre des femelles formées. Les larves L2 infectieuses présentes à l'état libre à un moment donné dans les racines constituent pour la plante le potentiel de nuisibilité qui sera analysé dans ce travail.

\section{MATÉRIEL ET MÉTHODES}

\section{A. Le nématode}

Du sol naturellement infesté par $M$. naasi est prélevé en janvier 1982 dans une parcelle du Rheu (Ille-etVilaine) cultivée en blé l'année précédente. Les densités de larves infectieuses (L2) y sont faibles et avoisinent 2 par $\mathrm{g}$ de sol.

\section{B. Le végétal}

Dix variétés de ray-grass inscrites au catalogue français des espèces et variétés cultivées sont retenues pour cette étude. Dans la pratique agricole de la région, les ray-grass anglais, Lolium perenne L.: GrasslandsRuani, Réveillé et Vigor sont capables de produire 5 ans au moins. Les ray-grass hybrides, Lolium $X$ hybridum Hauss : Augusta Dalita et Sabrina peuvent être maintenus 3 ans sur une même parcelle. Mais les ray-grass italiens, Lolium multiflorum Lam. présentent une pérennité moindre, soit environ 18 mois pour les variétés alternatives Billion et Promenade et 2 ans pour les cultivars non alternatifs Tiara et Tétrone.
Trois de ces variétés sont diploïdes: GrasslandsRuani, Vigor et Tiara, alors que les 7 autres sont tétra ploïdes.

\section{L'expérimentation}

Le sol infesté est mis dans des pots en terre cuite de $30 \mathrm{~cm}$ de diamètre, à raison de 2 répétitions par variété. Les pots sont conservés en conditions extérieures au Rheu (Ille-et-Vilaine) durant toute la période d'essai et sont posés à même le sol. Ils sont répartis en blocs de 2 pots correspondant pour chacun d'entre eux à une variété, sur une surface totale d'expérimentation de $3,9 \times 0,7 \mathrm{~m}$.

Les semis sont effectués le 29 avril 1982 avec des densités similaires à celles utilisées dans la pratique agricole, soit 87 ou 73 graines par pot correspondant à 20 ou $35 \mathrm{~kg} /$ ha pour les variétés diploïdes ou tétraploïdes respectivement.

En cours de culture chaque pot reçoit une fertilisation complète $\left(\mathrm{N}, \mathrm{P}_{2} \mathrm{O}_{5}, \mathrm{~K}_{2} \mathrm{O}\right)$ sous forme d'engrais liquide une fois par mois, à l'exception de la période hivernale allant de début novembre à fin février. L'arrosage est régulier durant l'été, pour éviter tout déficit hydrique. Des coupes sont réalisées tous les 2 mois à partir de début mai et jusqu'en novembre.

Des plantes de chacun des pots sont prélevées à l'aide d'un déplantoir, 7 fois au cours des 19 premiers mois de culture, soit en tout 14 échantillons par variété. Pour des raisons pratiques les systèmes radiculaires des plantes analysées ne peuvent être pris en totalité. Ils sont prélevés sur une profondeur variant entre 10 et $15 \mathrm{~cm}$ maximum et correspondant en général à un poids de racine allant de 6 à $16 \mathrm{~g}$ par échantillon.

Les prélèvements blessent très probablement les racines des plantes voisines et pour éviter au maximum les conséquences de ce traumatisme, nous ne prenons jamais 2 échantillons consécutifs dans la même partie du pot. Le trou réalisé dans le sol du pot après prélèvement est immédiatement comblé par l'apport d'un mélange sable-terreau préalablement stérilisé à la vapeur.

En fin de culture, un prélèvement de sol est effectué dans chaque pot à l'aide d'une gouge, soit 2 échantillons par variété, pour évaluer les populations de larves infectieuses (L2) qui y sont présentes.

\section{Les analyses nématologiques}

Les systèmes radiculaires des plantes prélevées sont lavés et les indices de galle sont notés pour chaque plante et correspondent à l'échelle suivante :

$\begin{array}{cc}\text { indices (i) } & \text { nombre de galles par plante } \\ 0 & 1 \text { à } 10 \\ 1 & 11 \text { à } 25 \\ 2 & 26 \text { à } 50 \\ 3 & 51 \text { à } 100 \\ 4 & \text { supérieur à } 100\end{array}$

Un indice pondéré d'abondance de galles est calculé pour chaque répétition de l'essai. Cet indice est défini 
en fonction du nombre variable $\mathrm{N}$ de plantes sur lequel ont porté les notations, des fréquences (fi) relatives aux indices (i) affectés à chaque plante et du nombre de classes d'indice i, $\mathrm{C}=6$ (CAUBEL et al., 1971).

Indice pondéré de galle $=\Sigma \mathrm{i} \times \mathrm{fi} \times 100 / \mathrm{N}(\mathrm{C}-1)=$ $=\Sigma \mathrm{i} \times \mathrm{fi} \times 100 / \mathrm{N} \times 5$.

Les racines sont ensuite coupées en morceaux de 1 à $2 \mathrm{~cm}$ de long et broyées durant 2 fois $30 \mathrm{~s}$ à la vitesse lente $(15000 \mathrm{t} / \mathrm{mn})$ d'un mixeur WARING.

Les nématodes sont extraits des racines par la méthode centrifugation-flottation avec une solution sucrée de densité 1,18 (COOLEN \& D'HERDE, 1972). Leurs effectifs sont déterminés au stéréomicroscope et rapportés au g de racine fraîche.

En fin de culture, les larves infectieuses (L2) sont extraites d'un échantillon de $100 \mathrm{~g}$ de sol par la méthode centrifugation-flottation déjà utilisée pour les racines. Leurs nombres sont rapportés au g de sol sec.

\section{E. L'analyse statistique}

Les différences enregistrées entre les variétés et les dates dans la formation des galles, femelles, mâles, œufs ou stades larvaires infectieux (L2) du nématode sont mises en évidence grâce à des analyses de variance à 2 facteurs (variété et date) faites sur l'ensemble des données acquises pour chacune des variables. Pour chaque variété, toutes les variables étudiées sont analysées sur l'ensemble des 7 prélèvements à l'exception de celles concernant les larves L2 qui ne prennent en compte que les 6 derniers d'entre eux. L'analyse statistique des résultats sera poursuivie, si nécessaire, par un test de DUNCAN pour préciser les différences notées pour chaque variable entre dates et variétés. Dans un but de simplification de présentation des résultats, nous exposerons successivement ceux obtenus par variété, puis par date. Nous signalerons et interpréterons les interactions existant entre les 2 facteurs. Toutes les données $(\mathrm{x})$, à l'exception des indices de galle, sont transformées en $\operatorname{Ln}(\mathrm{x}+1)$ dans le but d'homogénéiser les variances.

\section{RÉSULTATS}

L'évolution des populations du nématode au cours du temps est plus particulièrement étudiée sur les stades de son développement dépendant directement de l'hôte (femelles et œufs) ainsi que sur les larves infectieuses (L2) représentant un potentiel de nuisibilité. L'espèce pouvant se reproduire par parthénogenèse, l'étude de la production des mâles sera seulement abordée. On essaiera d'établir des corrélations entre la formation des galles et les autres éléments d'évaluation de l'intensité de l'attaque.

\section{A. Formation des femelles adultes}

Globalement, au cours des 19 premiers mois de culture les ray-grass italiens produisent significativement plus de femelles du nématode que les ray-grass anglais et hybrides (fig. 1 et tabl. 1). Parmi ces ray-grass italiens, les 2 variétés alternatives Promenade et Billion sont statistiquement les meilleurs hôtes pour le parasite. Les ray-grass anglais, à l'exception de GrasslandsRuani hôte le plus médiocre de $M$. naasi, produisent des populations femelles équivalentes à celles des raygrass hybrides (fig. 1 et tabl. 1).

Des nombres plus élevés de femelles sont trouvés en fin d'été ou en fin d'automne avec des maxima enregistrés en décembre lors de la $1^{\text {re }}$ années de culture et plus précocément en septembre ou novembre suivant la variété, la $2^{\mathrm{e}}$ année (fig. 1 et tabl. 2).

Une interaction significative au seuil 0,01 , notée entre les facteurs variété et date, serait sans doute en partie le reflet de la formation des populations maximales de femelles sur ray-grass italiens de type alternatif dès décembre 1982, les autres ray-grass ne les formant qu'à partir de novembre 1983 (fig. 1).

\section{B. Formation des mâles adultes}

La production de mâles est très faible chez les raygrass anglais et hybrides et plus particulièrement chez Grasslands-Ruani, hôte le plus médiocre du nématode (tabl. 1). Elle reste dans tous les cas inférieure à 4 mâles par g de racine (tabl. 1 et 2). A l'inverse les populations de mâles sont plus élevées chez 1 s variétés de ray-grass italiens produisant le plus de femelles de M. naasi (tabl. 1).

Pour chaque prélèvement la production des mâles, toutes variétés confondues, est corrélée positivement à celle des femelles (tabl. 3).

\section{Production des aufs}

Le nombre d'œufs formés par $\mathrm{g}$ de racine dépend dans l'absolu de la fécondité des femelles et de l'effectif des populations femelles présentes dans les racines.

\section{Fécondité des femelles}

La fécondité des femelles ne diffère pas statistiquement chez 8 des 10 variétés prises en compte. Globalement, elle est plus importante en fin d'été ou en fin d'automne (tabl. 2).

Une interaction significative au seuil 0,01 est notée entre les facteurs variété et date. La fécondité des femelles de chaque variété est globalement la même sur toute la période de l'essai, mais elle peut cependant différer pour une date donnée d'un cultivar à un autre (tabl. 1 et 2).

\section{Nombre d'œufs produits par g de racine}

Les 2 variétés de ray-grass anglais Grasslands-Ruani et Vigor fournissent beaucoup moins d'œufs que les 8 autres cultivars testés (fig. 2, tabl. 1). La production d'œufs des ray-grass italiens n'est pas souvent significativement süpérieure à celles des 3 ray-grass hybrides ou du ray-grass anglais cv. Réveillé. Ces 4 ray-grass italiens forment cependant en moyenne le plus d'œufs par g de racines. Pour un cultivar donné, les valeurs extrêmes enregistrées parmi les nombres d'œufs produits par $g$ de racine sont très souvent éloignées et 


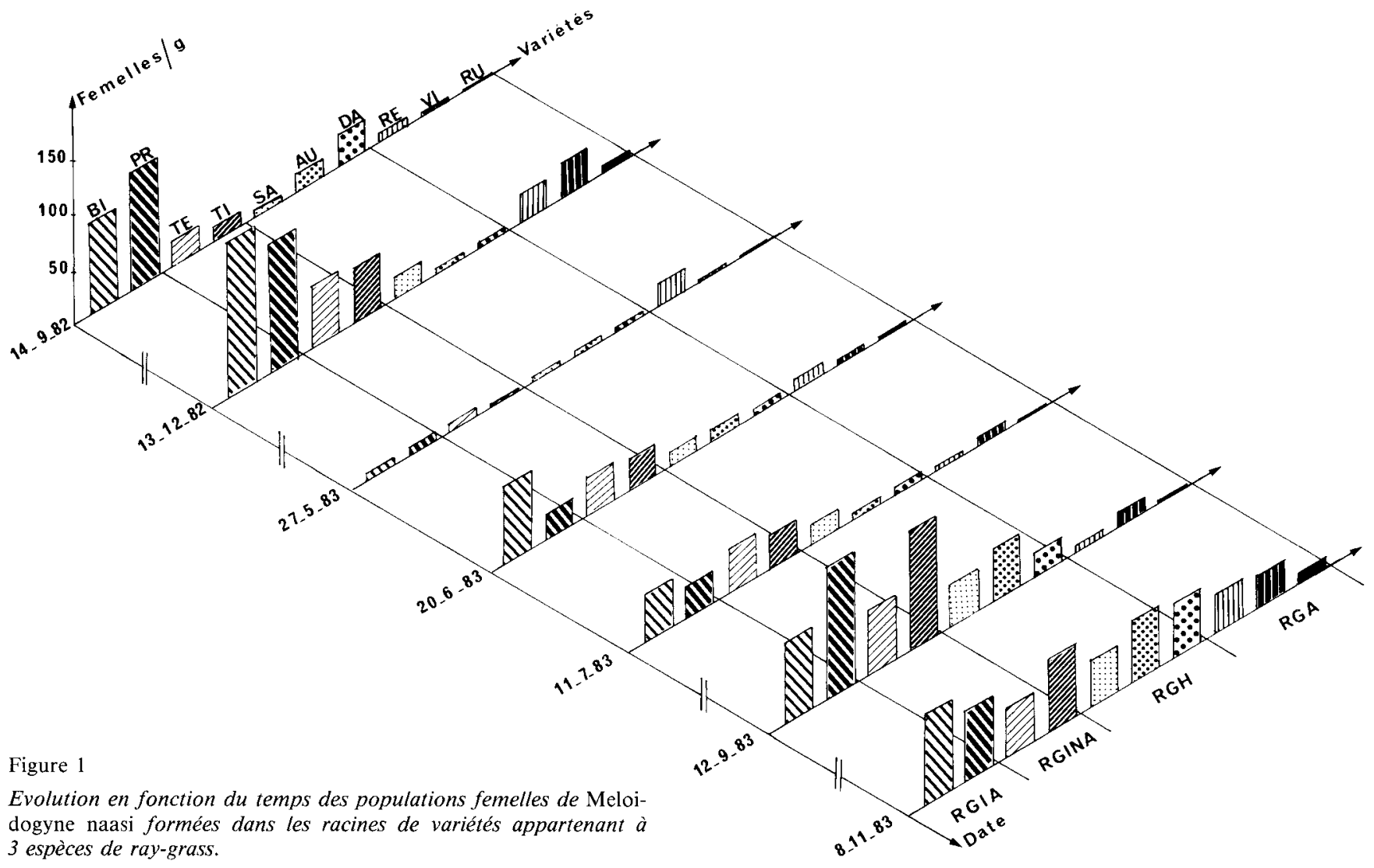

$B I:$ Billion $\}$ ray-grass italien alternatif

$P R$ : Promenade $\}$ (alternative italian ryegrass)

TE : Tétrone \} ray-grass italien non alternatif

$T I$ : Tiara $\}$ (non-alternative italian ryegrass)

$R G I A$

$S A$ : Sabrina

$\left.\begin{array}{l}A U: \text { Augusta } \\ D A: \text { Dalita }\end{array}\right\}$ ray-grass hybride (hybrid ryegrass)

RGINA

RE : Réveillé

$\left.\begin{array}{l}V I: \text { Vigor } \\ R U: \text { Ruani }\end{array}\right\}$ ray-grass anglais (perennial ryegrass) $R G A$

$R G H$

Relationships between the female populations of Meloidogyne naasi found in the roots of ryegrass varieties and the experiment time.

TABLEAU I

Etude statistique de l'influence du facteur variétal sur la formation des galles et les populations de Meloidogyne naasi dans des racines de ray-grass.

- - données transformées en $\operatorname{Ln}(x+1)$.

- moyennes calculées sur 7 ou $6^{\times}$dates,

- les valeurs affectées par les mêmes lettres ne diffèrent pas entre elles après l'analyse au test de Duncan au seuil 0,05,

- $g: g$ de racine.

Statistical analysis of the influence of variety factor on the production of galls and populations of Meloidogyne naasi on the roots of ryegrasses.

- transformation of data: $\operatorname{Ln}(x+1)$

- mean of 7 or $6^{\times}$dates,

- values with the same letter are not significantly different according to Duncan test at $P=0.05$,

$-g: g$ of root.

\begin{tabular}{|c|c|c|c|c|c|c|}
\hline Variétés & - femelles & $\begin{array}{l}\bullet \text { œufs } \\
\text { /femelle }\end{array}$ & 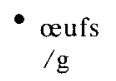 & $\begin{array}{l}\mathrm{L} 2^{\times} \\
/ \mathrm{g}\end{array}$ & - mâles & $\begin{array}{l}\text { Indice de } \\
\text { galle }\end{array}$ \\
\hline \multicolumn{7}{|l|}{ Ray-grass italien } \\
\hline \multicolumn{7}{|l|}{ Alternatif } \\
\hline Billion & $3,96^{\mathrm{a}}$ & $4,21^{\mathrm{abc}}$ & $8,13^{a}$ & $4,67^{\mathrm{abc}}$ & $1,17^{\mathrm{b}}$ & $63,8^{\mathrm{bc}}$ \\
\hline Promenade & $3,91^{\mathrm{a}}$ & $3,86^{b c}$ & $7,72^{\mathrm{abc}}$ & $4,68^{\mathrm{abc}}$ & $1,75^{\mathrm{a}}$ & $74,6^{\mathrm{a}}$ \\
\hline \multicolumn{7}{|l|}{ Non alternatif } \\
\hline Tétrone & $3,43^{b}$ & $4,52^{\mathrm{ab}}$ & $7,88^{\mathrm{ab}}$ & $5,05^{\mathrm{ab}}$ & $1,17^{\mathrm{b}}$ & $67,1^{\mathrm{abc}}$ \\
\hline Tiara & $3,35^{\mathrm{b}}$ & $4,52^{\mathrm{abc}}$ & $7,66^{\mathrm{abc}}$ & $4,93^{\mathrm{ab}}$ & $1,59^{\mathrm{ab}}$ & $71,1^{\mathrm{ab}}$ \\
\hline \multicolumn{7}{|l|}{ Ray-grass hybride } \\
\hline Sabrina & $2,71^{\mathrm{c}}$ & $4,77^{\mathrm{a}}$ & $7,37 \mathrm{abc}$ & $5,06^{\mathrm{a}}$ & $0,54^{\mathrm{c}}$ & $65,1^{b c}$ \\
\hline Augusta & $2,68^{c}$ & $4,60^{\mathrm{a}}$ & $7,17^{b c}$ & $4,74^{\mathrm{abc}}$ & $0,39^{\text {cd }}$ & $62,4^{b c}$ \\
\hline Dalita & $2,47^{\mathrm{cd}}$ & $4,62^{\mathrm{a}}$ & $6,94^{c}$ & $4,20^{\mathrm{bc}}$ & $0,39^{\text {cd }}$ & $60,1^{\mathrm{c}}$ \\
\hline \multicolumn{7}{|l|}{ Ray-grass anglais } \\
\hline Réveillé & $2,71^{\mathrm{c}}$ & $4,54^{\mathrm{a}}$ & $7,15^{\mathrm{bc}}$ & $4,49^{a b c}$ & $0,51^{\mathrm{cd}}$ & $66,4^{\mathrm{abc}}$ \\
\hline Vigor & $2,08^{d}$ & $4,14^{\mathrm{abc}}$ & $6,19 \mathrm{~d}$ & $4,02^{\mathrm{c}}$ & $0,13^{\mathrm{d}}$ & $58,0^{\mathrm{c}}$ \\
\hline Grassland-Ruani & $0,99 \mathrm{e}$ & $3,73^{c}$ & $4,39^{\circ}$ & $2,86^{\mathrm{d}}$ & $0,06^{\mathrm{d}}$ & $49,4^{\mathrm{d}}$ \\
\hline
\end{tabular}


TABLEAU 2

Etude statistique de l'influence du facteur date sur la formation des galles et des populations de Meloidogyne naasi dans des racines de ray-grass.

- données transformées en Ln $(x+1)$,

- moyenne calculée sur 10 variétés,

- les valeurs affectées par les mêmes lettres ne diffèrent pas entre elles après analyse au test de Duncan au seuil 0,05,

$-g: g$ de racine.

Statistical analysis of the influence of date factor on the production of galls and populations of Meloidogyne naasi on the roots of ryegrass.

- transformation of data: $\operatorname{Ln}(x+I)$.

- mean of 10 varieties,

- values with the same letter are not significantly different according to Duncan test at $P=0.05$,

$-g: g$ of root.

\begin{tabular}{|c|c|c|c|c|c|c|}
\hline Dates & $\begin{array}{c}\text { - femelles } \\
/ \mathrm{g}\end{array}$ & $\begin{array}{c}\text { - œufs } \\
\text { /femelle }\end{array}$ & $\begin{array}{l}\text { oufs } \\
/ \mathrm{g}\end{array}$ & $\begin{array}{l}\text { L2 } \\
/ \mathrm{g}\end{array}$ & - ${ }_{/ \mathrm{g}}^{\mathrm{g}}$ & $\begin{array}{l}\text { Indice de } \\
\text { galle }\end{array}$ \\
\hline 14- $9-82$ & $2,83^{b}$ & $4,09^{\circ}$ & $6,72^{\mathrm{c}}$ & - & $0,52^{\mathrm{ab}}$ & 41,3 \\
\hline $13-12-82$ & $3,25^{\mathrm{a}}$ & $4,64^{a b}$ & $7,81^{b}$ & $6,24^{b}$ & $0,76^{\mathrm{ab}}$ & $50,3^{d}$ \\
\hline $27-5-83$ & $1,64^{c}$ & $4,00^{\mathrm{dd}}$ & $5,88^{\mathrm{d}}$ & $4,31^{d}$ & $0,21^{\mathrm{c}}$ & $78,0^{\mathrm{a}}$ \\
\hline $20-6-83$ & $2,58^{b}$ & $3,52^{\mathrm{d}}$ & $5,96^{d}$ & $3,96^{\mathrm{d}}$ & $1,07 \mathrm{a}$ & $76,0^{\mathrm{ab}}$ \\
\hline $11-7-83$ & $2,59^{\mathrm{b}}$ & $4,24^{b c}$ & 6,64 & $4,48^{d}$ & $0,86^{\mathrm{ab}}$ & $63,0^{c}$ \\
\hline $8-11-83$ & $3,63^{\mathrm{a}}$ & $5,09^{\mathrm{a}}$ & $8,55^{a}$ & $6,88^{\mathrm{a}}$ & $1,12^{\mathrm{a}}$ & $70,0^{\mathrm{bc}}$ \\
\hline
\end{tabular}

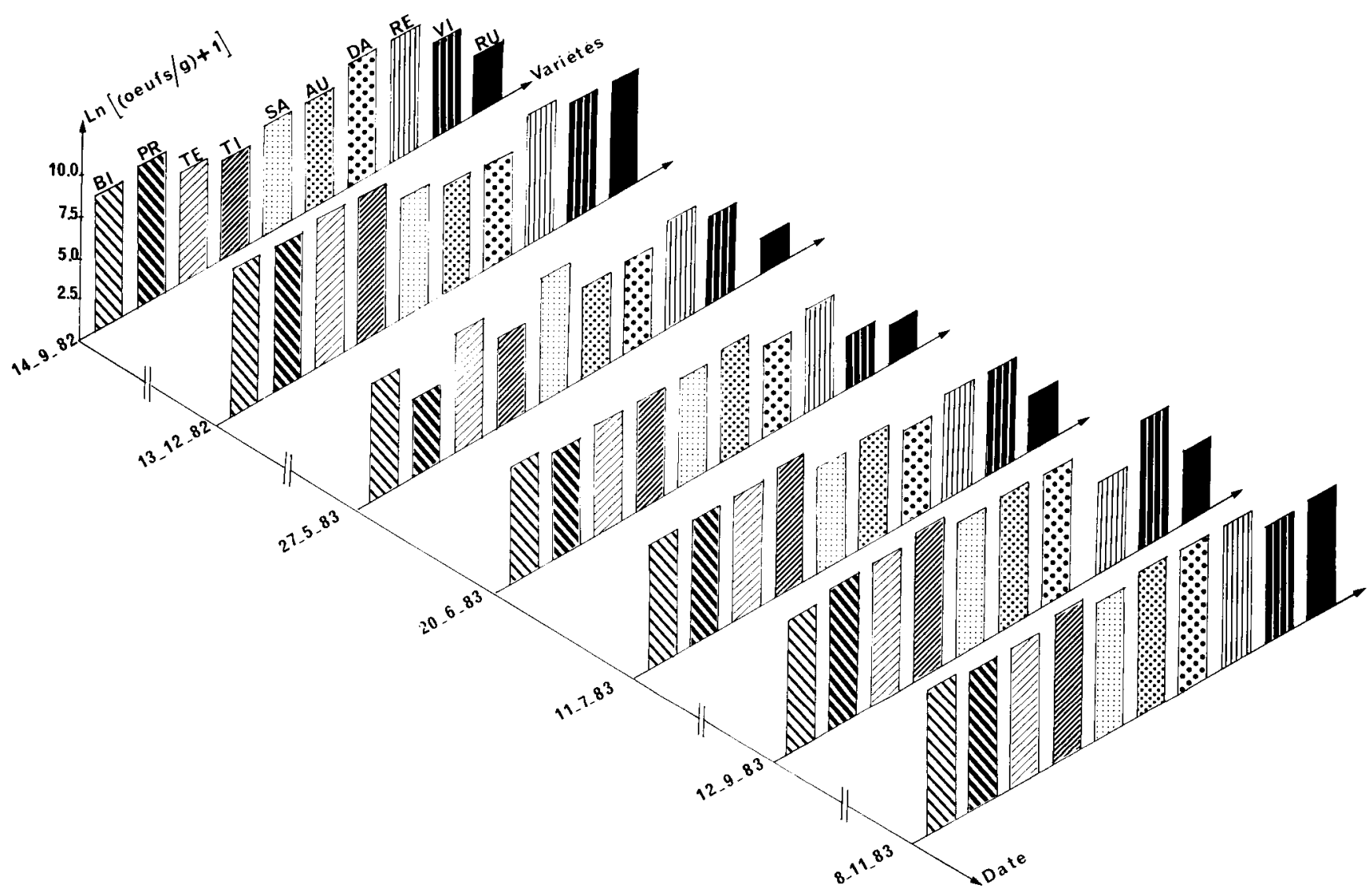

Figure 2

Evolution en fonction du temps des nombres d'œufs de Meloidogyne naasi formés dans les racines de variétés appartenant à 3 espèces de ray-grass.

sont à l'origine d'écarts types assez grands. Dans ce cas, l'analyse de variance ne différencie plus la production des œufs d'un cultivar à l'autre.

La production des œufs évaluée par g de racine reste plus importante en automne et en début de période hivernale avec un maximum enregistré en fin d'essai le 8-11-83 (fig. 2 et tabl. 2).

Une interaction significative au seuil 0,05 , notée
Relationships between the eggs numbers of Meloidogyne naasi found in the roots of ryegrass varieties and the experiment time.

entre les facteurs variété et date, serait sans doute liée à la plus grande productivité en oufs des ray-grass italiens comparée à celles des 2 autres espèces de ray-grass lors des prélèvements faits en décembre 1982 uniquement (fig. 2).

Pour chaque prélèvement, le nombre d'œufs formés par g de racine, toutes variétés confondues, est corrélé positivement à l'abondance des femelles (tabl. 3). 
TABLEAU 3

Etude des corrélations existant entre la formation des galles et lou les divers stades de développement de Meloidogyne naasi sur 10 variétés de ray-grass.

- coefficient de corrélation significatif au seuil $0,05^{*}, 0,01^{* *}$ et $0,001^{* * *}$,

- 19 degrés de liberté,

- $g: g$ de racine.

Study of the correlation between the production of galls and (or) different development stages of Meloidogyne naasi on 10 ryegrass varieties.

$$
\begin{aligned}
& \text { - correlation coefficient significant at } P=0.001^{* * *}, \\
& P=0.01^{* *}, P=0.05^{*}, \\
& -19 \text { degrees of freedom, } \\
& -g: g \text { of root. }
\end{aligned}
$$

\begin{tabular}{|c|c|c|c|c|}
\hline \multirow{2}{*}{ Dates } & \multicolumn{4}{|c|}{$\begin{array}{c}\text { Coefficient de corrélation } \\
r \text { entre }\end{array}$} \\
\hline & $\begin{array}{c}\text { mâles et } \\
\text { femelles } \\
/ \mathrm{g}\end{array}$ & $\begin{array}{c}\text { œufs et } \\
\text { femelles } \\
/ g\end{array}$ & $\begin{array}{c}\text { L2 et } \propto u f s \\
/ g\end{array}$ & $\begin{array}{l}\text { Indice de } \\
\text { galle et } \\
\text { femelles } / \mathrm{g}\end{array}$ \\
\hline $14-9-82$ & $0,562^{* *}$ & $0,891^{* * *}$ & - & $0,500^{*}$ \\
\hline $13-12-82$ & $0,849 * * *$ & $0,928^{* * *}$ & $0,893 * * *$ & $0,760 * * *$ \\
\hline $27-5-83$ & $0,612^{* *}$ & $0,617^{* *}$ & $0,906^{* * *}$ & - \\
\hline $20-6-83$ & $0,742 * * *$ & $0,823^{* * *}$ & $0,537^{*}$ & $0,524^{*}$ \\
\hline $11-7-83$ & $0,690^{* * *}$ & $0,896 * * *$ & $0,890^{* * *}$ & $0,637^{* *}$ \\
\hline $12-9-83$ & $0,649^{* *}$ & $0,920 * * *$ & $0,637 * *$ & $0,646 * *$ \\
\hline $8-11-83$ & $0,726 * * *$ & $0,849^{* * *}$ & $0,453^{*}$ & $0,542 *$ \\
\hline
\end{tabular}

\section{Production de larves infectieuses L2}

Les populations de larves infectieuses $\mathbf{L} 2$ ont été suivies régulièrement dans les racines tout le long de l'essai. Une seule analyse a concerné les populations de L2 présentes dans le sol. Elle a été réalisée à la fin de l'essai.

\section{Etude des populations $L 2$ endoradiculaires par $g$ de racine}

Le ray-grass hybride cv. Sabrina fournit le plus de larves L2, mais leurs nombres ne diffèrent pas significativement de ceux enregistrés chez les 6 autres variétés appartenant aux ray-grass hybride, anglais et italien. Grasslands-Ruani puis Vigor restent les cultivars qui donnent statistiquement le moins de larves (fig. 3 et tabl. 1). Il est par contre difficile de dissocier entre elles les productions de larves sur les 8 autres cultivars.

Les périodes hivernales sont celles durant lesquelles les populations larvaires sont les plus fortes. Leur maximum est rencontré au cours de l'hiver de la $2^{\mathrm{e}}$ année de culture (fig. 3, tabl. 2).

Pour chaque prélèvement, le nombre de $\mathrm{L} 2$ par g de racine est corrélé positivement à celui des œufs (tabl. 3). La plupart des L2, doivent être des larves «enchorionnées » dont la sortie a été provoquée pré-

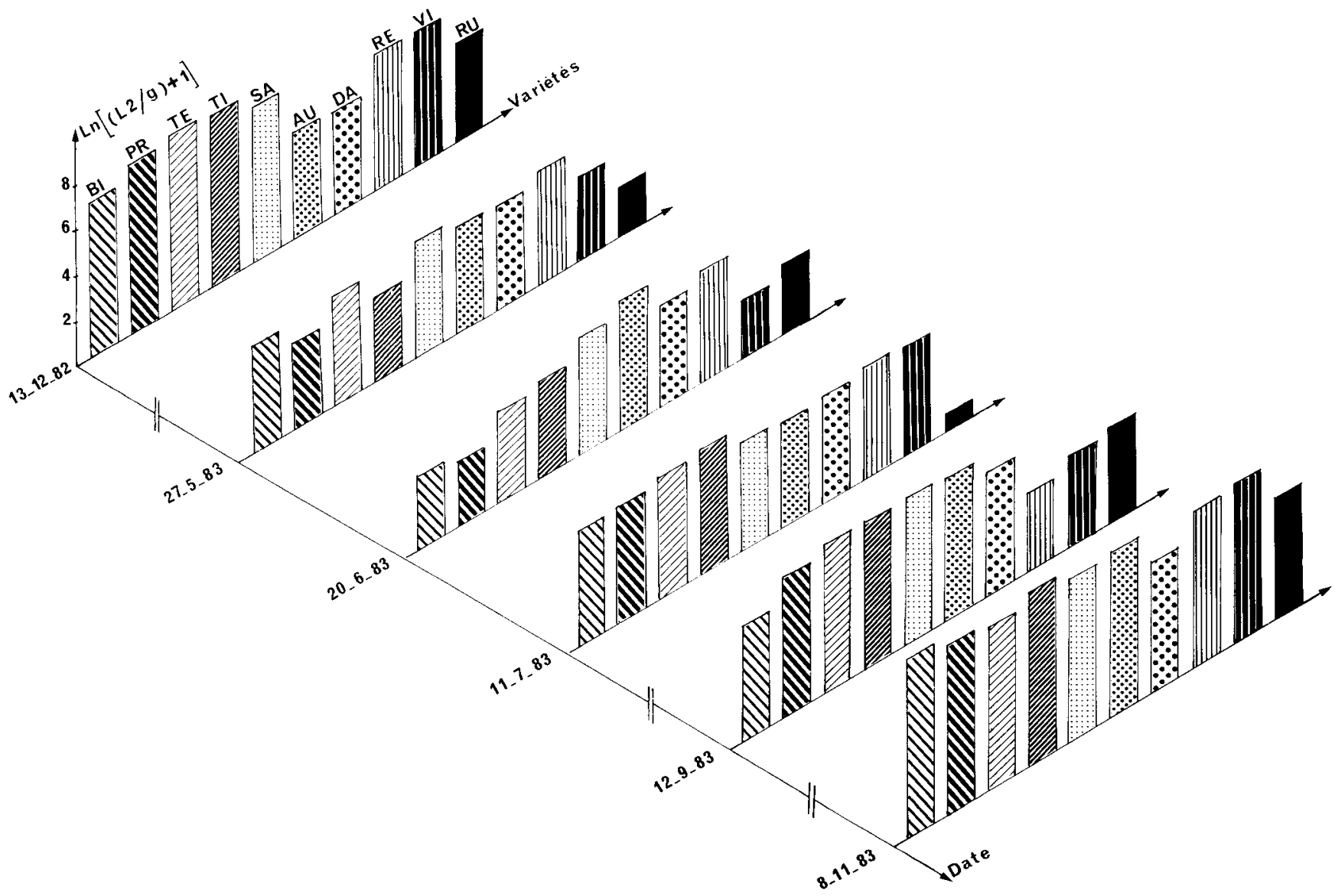

Figure 3

Evolution en fonction du temps des populations larvaires $L 2$ de Meloidogyne naasi formées dans les racines de variétés appartenant à 3 espèces de ray-grass.
Relationships between the larvae population L2 of Meloidogyne naasi found in the roots of ryegrass varieties and the experiment time. 
maturément de l'œuf par l'extraction car leur nombre est proportionnel à celui des œufs trouvés par g de racine.

\section{Etude des populations L2 du sol}

Après 2 ans de culture les populations $\mathrm{L} 2 \mathrm{du}$ sol sont faibles et leur maximum avoisine $5 \mathrm{~L} 2$ par $\mathrm{g}$ de sol (tabl. 4). Les pots cultivés avec les ray-grass italiens de type alternatif, cvs Billion et Promenade, présentent les populations L2 les plus faibles statistiquement. A cette période de l'essai, seules les parties aériennes des plantes de ces 2 variétés sont totalement desséchées. Il est par ailleurs impossible de distinguer des différences entre les nombres de $\mathrm{L} 2$ trouvées chez les autres graminées testées, dont l'état végétatif reste bon.

\section{TABLEAU 4}

Etude statistique des populations larvaires $L 2$ de Meloidogyne naasi trouvées dans le sol en fin d'essai réalisé sur variétés de ray-grass.

\section{- $m$ = moyenne,}

- $p=$ valeurs extrêmes minimale ou maximale,

- les valeurs affectées par les mêmes lettres ne diffèrent pas entre elles après analyse au test de Duncan au seuil 0,05.

Statistical analysis of $L 2$ larvae populations of Meloidogyne naasi found in the soil at the end of the experiment concerning ryegrass varieties.

$-m=$ mean,

$-p=$ maximum and minimum values,

- values with the same letter are not significantly different according to Duncan test at $P=0.05$.

\begin{tabular}{lcc}
\hline \hline & $\mathrm{m}$ & $\mathrm{p}$ \\
\hline Ray-grass italien alternatif & $0,57^{\mathrm{b}}$ & $(0,13-0,88)$ \\
Ray-grass italien non alternatif & $3,37^{\mathrm{a}}$ & $(1,58-5,49)$ \\
Ray-grass hybride & $2,94^{\mathrm{a}}$ & $(0,49-3,99)$ \\
Ray-grass anglais & $2,14^{\mathrm{a}}$ & $(0,75-2,36)$ \\
\hline
\end{tabular}

\section{E. Formation des galles}

L'analyse des indices de galle ne permet pas de dissocier les variétés. En particulier, elle ne permet pas de distinguer le groupe des ray-grass italiens comme étant de meilleurs hôtes que les autres ray-grass. Seule la variété cv. Grasslands-Ruani, hôte le plus médiocre de $M$. naasi, peut être détectée après contrôle visuel des galles (tabl. 1).

A l'exception du prélèvement effectué en mai 1985, période durant laquelle les populations femelles toutes variétés confondues sont les plus faibles, les indices de galle sont corrélés à l'abondance des femelles (tabl. 3). Ce résultat n'est pas surprenant, car la formation des galles est considérée comme étant indispensable au développement du nématode jusqu'au stade femelle adulte.

\section{DISCUSSION ET CONCLUSION}

L'influence de l'âge de la culture va être analysée successivement par ses effets sur les qualités d'hôte des différentes espèces de graminées, et sur la fluctuation des populations endoradiculaires du nématode. Des conséquences pratiques pourront alors être tirées.

\section{A. Evolution des qualités d'hôte des ray-grass en fonc- tion de l'âge de la culture}

Avec les variétés testées, nous obtenons au cours des 19 premiers mois de culture, une formation des femelles fonction de l'espèce ou de la variété de ray-grass considérée. Les ray-grass italiens donnent les nombres de femelles formées par $\mathrm{g}$ de racine les plus élevés, les variétés alternatives étant les meilleurs hôtes. Les qualités d'hôte des 3 ray-grass hybrides et du ray-grass anglais cv. Réveillé sont similaires. Les variétés de raygrass anglais cvs Vigor et Grasslands-Ruani restent toujours des hôtes médiocres. Ce classement correspond à celui effectué en 1982 après 4 mois de culture et une seule génération du nématode (PERSON-DEDRYVER \& FISCHER, 1987). La qualité intrinsèque d'hôte ne change pas au cours de tout l'essai et le temps ne fait qu'accentuer globalement les différences entre les raygrass italiens et les autres graminées. Les qualités d'hôte médiocre de cvs Vigor et Grasslands-Ruani correspondent sans doute à des caractéristiques variétales des ray-grass anglais et sont la preuve de l'existence d'une variabilité intraspécifique dans les relations "hôte-parasite". Mais globalement le nombre de femelles formées semble d'autant plus élevé que l'espèce de graminée est moins persistante. En effet, la pérennité des ray-grass anglais et hybrides est bien supérieure à celles des ray-grass italiens, dont les variétés de type alternatif sont particulièrement peu persistantes. Il paraît difficile de donner une explication à ce phénomène. Nous pouvons juste signaler que JACKSON \& ROY (1986) démontrent que la vitesse de croissance et la biomasse des feuilles sont plus importantes chez les graminées annuelles que chez les pérennes. Cette vitesse de croissance supérieure des ray-grass italiens pourrait favoriser le développement du nématode en femelle.

La fécondité des femelles n'est pas affectée par la nature des relations «plante-parasite » : elle est statistiquement identique pour la plupart des cultivars. Grasslands-Ruani est la variété qui induit la fécondité la moins importante des femelles. Les fécondités n'étant pas très différentes d'un cultivar à un autre, les nombres d'œufs trouvés par $\mathrm{g}$ de racine sont essentiellement le reflet de la densité des femelles, comme le prouvent les corrélations positives entre les nombres d'œufs et de femelles par $g$ de racine. Chez les raygrass anglais Grasslands-Ruani et Vigor, le faible nombre d'œufs correspond à la fois à un petit effectif de femelles formées par $\mathrm{g}$ de racine et à une baisse de fécondité.

Les populations de $\mathrm{L} 2$ correspondent, soit à des larves écloses et présentes dans les racines au moment du prélèvement, soit à des larves « enchorionnées ». Deux cultivars ressortent de l'analyse cv. Grasslands-Ruani présentant le moins de nématodes au stade $\mathbf{L} 2 \mathrm{et} \mathrm{cv}$. Sabrina en produisant le plus. Il n'existe pas de différences entre les autres cultivars.

\section{B. Evolution de l'importance des populations endora- diculaires en fonction de l'âge de la culture}

Les courbes d'évolution des populations femelles (fig. 1) mettent très bien en évidence l'apparition de 2 générations annuelles de $M$. naasi sur ray-grass. La 
$1^{\text {re }}$ génération se forme en juin et est surtout visible chez les ray-grass italiens le 20-6-83. Une $2^{\mathrm{e}}$ génération est possible en automne, comme l'ont signalé FRANKLIN et al. (1971). Elle est bien visible le 13-12-82 lors de la $1^{\text {re }}$ année de culture et plus précocement, à partir de la mi-septembre 1983 , lors de la $2^{\mathrm{e}}$ année de culture. Le maximum des populations femelles est alors atteint plus rapidement chez les ray-grass italiens (12-9-83) que chez les ray-grass anglais et hybrides (8-11-83). Le ray-grass italien favorise un développement plus rapide du nématode que les autres graminées testées. Ce phénomène a déjà été signalé après étude de la génération printanière du nématode sur un ray-grass italien $\mathrm{cv}$. Promenade et une fétuque élevée cv. Pastelle (PERSONDEDRYVER \& FISCHER, 1987).

Chez les ray-grass anglais et hybrides, c'est la dernière génération formée au cours de l'essai qui produit le plus de femelles, alors que la population est d'emblée importante chez les ray-grass italiens. Cette constatation démontre une fois de plus que les raygrass italiens favorisent le développement du nématode. Les cultures de ray-grass anglais et hybrides augmentent progressivement les potentiels infectieux du nématode au cours du temps (fig. 2 et 3 ).

\section{Utilisations des données acquises}

Ce travail apporte des renseignements sur les périodes les plus propices pour effectuer des prélèvements et des analyses nématologiques sur ray-grass. L'automne paraît être la saison qui correspond au développement maximum de $M$. naasi dans les racines de graminées et les prélèvements qui y sont réalisés donnent une bonne idée de l'importance des populations du parasite permettant de prévoir les dégâts à venir. Pour des raisons inverses, des analyses au printemps doivent être évitées.
La seule observation des galles ne peut en aucun cas être un bon critère d'analyse đu caractère hôte des raygrass. Il convient de la compléter par l'étude des populations endoradiculaires du nématode.

La détection de la qualité d'hôte très médiocre du ray-grass anglais cv. Grasslands-Ruani peut être mise à profit, en introduisant cette variété dans les rotations, lorsque les conditions économiques imposent la culture de ray-grass. Parmi les cultivars testés cv. GrasslandsRuani est en effet le seul susceptible de limiter sérieusement le développement de $M$. naasi.

Les bonnes potentialités d'hôte des ray-grass, déjà signalées sur une courte période (PERSON-DEDRYVER \& FISCHER, 1987) se maintiennent en cours de culture. Les conséquences de l'utilisation de ray-grass dans les rotations sont, de toute évidence, une augmentation rapide des populations du déprédateur dangereuse pour les cultures suivant la graminée. Cependant, 2 facteurs peuvent affecter le caractère bon hôte des ray-grass : leur manque de pérennité et la sensibilité de la plante à la présence du nématode.

En effet, quand la culture arrive à sa limite de production, les populations du nématode dans le sol chutent, comme en témoignent nos résultats enregistrés sur ray-grass italien alternatif 19 mois après semis (tabl. 4). Ces populations L2 sont en effet plus faibles sur ray-grass italien que celles induites par les ray-grass anglais et hybrides plus persistants mais moins bon hôtes du nématode.

Par ailleurs, de fortes populations endoradiculaires de $M$. naasi peuvent endommager plus fortement certaines variétés moins tolérantes vis-à-vis de ce parasite et qui par conséquent multiplieront moins le nématode. C'est le cas du ray-grass italien cv. Promenade (PERSON-DEDRYVER et al., 1987).

Reçu le 24 juillet 1986. Accepté le 14 septembre 1987.

\section{RÉFÉRENCES BIBLIOGRAPHIQUES}

Caubel G., Lemaire J. M., Rivoal R., 1971. Observations préliminaires sur l'attaque de diverses céréales par Meloidogyne naasi en présence d'Heterodera avenae et d'Ophiobolus graminis. Conférences sur les nématodes du genre Meloidogyne. O.E.P.P., Antibes, 2729 octobre $1971,10 \mathrm{pp}$.

Cook R., York P. A., 1979. Nematodes and herbage improvement. Annu. Rep. Welsh Plant Breed. St., 177-207.

Cook R., York P. A., Evans J. L., Chew B. H., 1980. Intravarietal variation in grasses to Meloidogyne naasi. Annu. Rep. Welsh Plant Breed. St., 84-85.

Cook R., York P. A., 1983. Population dynamics and control of root-knot nematode. Annu. Rep. Welsh Plant Breed. St., 94-97.

Coolen W. A., D'Herde C. J., 1972. A method for the quantitative extraction of nematodes from plant tissue. Publ. Gov. Res. St. Nematol. Entomol., Merelbecke, Belgium, 1-77.

Franklin M. T., Clark S. A., Course J. A., 1971. Population chan- ges and development of Meloidogyne naasi in the field. Nematologica, 17, 575-590.

Gooris J., D'Herde C. J., 1977. Study on the biology of Meloidogyne naasi Franklin 1965. Publ. Gov. Res. St. Nematol. Entomol., Merelbecke, Belgium, 1-115.

Jackson L. E., Roy J., 1986. Growth patterns of mediterranean annual and perennial grasses under simulated rainfall regimes of southern France and California. Acta Oecologica, Oecologica Plantarum, 191-212.

Person-Dedryver F., Fischer J., 1987. Grasses as hosts of Meloidogyne naasi Franklin. I. Variation in host status of species and varieties grown in France. Nematologica, 33, 61-71.

Person-Dedryver F., Gueye P. S., Boulch M. F., 1987. Grasses as hosts of Meloidogyne naasi Franklin. II. Study of nematode populations and their effects on the yield of three varieties. Nematologica, $33,72-82$. 